\title{
Assessing The Performance Characteristics of Handheld Ultrasound in A Rheumatic Heart Disease Screening Program
}

\section{Zachary P. Kaltenborn ( $\sim$ Kalt0020@umn.edu )}

University of Minnesota

\section{Zewde. Anteneh}

University of Minnesota

Jonathan D. Kirsch

University of Minnesota

\section{Michelle Yates}

Soddo Christian Hospital

Katelyn M. Tessier

University of Minnesota

Eileen Nemec

Children's Hospitals and Clinics of Minnesota

\section{Ronald A. Johannsen}

Hennepin County Medical Center

\section{Research Article}

Keywords: handheld ultrasound, rheumatic heart disease, Lumify

Posted Date: June 16th, 2021

DOl: https://doi.org/10.21203/rs.3.rs-580374/v1

License: (9) (i) This work is licensed under a Creative Commons Attribution 4.0 International License. Read Full License 


\section{Abstract \\ Background}

Rheumatic heart disease affects 33 million people in low and middle income countries and is the leading cause of cardiovascular death among children and young adults. Penicillin prophylaxis has been shown to improve valvular function among patients with clinically silent or mild disease. Efforts to expand echocardiographic screening are focusing on simplified protocols, non-physician ultrasonographers, and portable ultrasound devices, including handheld ultrasound. Recent advances support the use of singleview screening protocols. With the increasing availability and low cost of handheld devices, prospective studies are needed to evaluate their performance in these settings.

\section{Methods}

We conducted a cross-sectional study among 19 at-risk school-children participating in a rheumatic heart disease screening program in Ethiopia comparing a handheld ultrasound device (Phillips Lumify) to a fully-equipped portable ultrasound machine (Sonosite M-Turbo).

\section{Results}

Agreement between devices was similar for expert and non-expert review (84\%). However, when reviewed by a non-expert the Lumify identified fewer screen-positive cases ( $p$-value 0.083 ). We also compared nonexpert to expert interpretation by device and found a significant difference in interpretation for the Lumify ( $p$-value 0.025). There was a trend towards shorter jet length by color Doppler in the handheld ultrasound device for both expert and non-expert review.

\section{Conclusions}

Our study highlights the importance of using caution when applying proposed single-view criteria as the sensitivity and specificity may be affected by the device.

\section{Background}

Rheumatic heart disease affects 33 million people in low and middle income countries and is the leading cause of cardiovascular death among children and young adults. ${ }^{1}$ Rheumatic heart disease also has substantial economic consequences and early identification could help to narrow disparities within affected populations. ${ }^{2,3}$ Rheumatic heart disease develops as a consequence of acute rheumatic fever which primarily affects children and will lead to rheumatic heart disease in $60 \%$ of cases. ${ }^{4}$ Recurrent attacks of acute rheumatic fever accelerate the progression of valvular damage and secondary 
prophylaxis with monthly penicillin is an effective strategy to improve survival. ${ }^{5-7}$ Penicillin prophylaxis has been shown to improve valvular function among patients with clinically silent or mild disease. ${ }^{8}$ Echocardiography has become the standard of care for detecting rheumatic heart disease as it can identify ten times the number of affected children when compared to physical exam. ${ }^{9}$ Echocardiographic screening of school-aged children in low and middle income countries has demonstrated a large burden of early rheumatic heart disease thus providing a critical time period for early intervention with penicillin and the prevention of rheumatic heart disease related deaths. ${ }^{10}$ In 2012, the World Heart Federation updated their guideline for the diagnosis of asymptomatic or latent rheumatic heart disease including criteria for 'definite' and 'borderline' disease. ${ }^{11}$ Using this guideline, cross-sectional echocardiographic studies have found a substantial burden of disease around the globe. ${ }^{12-16}$

While the World Heart Federation criteria serve as the gold standard for making a diagnosis of rheumatic heart disease, they are impractical for population-based screening programs due to time, cost, and resource availability. ${ }^{17}$ Efforts to expand echocardiographic screening are focusing on simplified protocols, non-physician ultrasonographers, and portable ultrasound devices, including handheld ultrasound. ${ }^{18}$ For example, Beaton et al. demonstrated that pediatric cardiologists employing a simplified handheld ultrasound protocol can achieve high diagnostic accuracy. ${ }^{19}$ However, shortages of echocardiographers and cardiologists in many endemic regions limit the expansion of population-based screening programs. ${ }^{20}$ Efforts to expand the screening responsibilities to include a non-physician workforce could overcome this limitation. ${ }^{21}$ Through focused training, non-physician ultrasonographers using simplified screening protocols can achieve a high sensitivity and specificity for detecting early rheumatic heart disease. ${ }^{22-25}$

Studies evaluating single image protocols with color Doppler of the mitral and aortic valves have demonstrated sensitivity of $73-92 \%$ and specificity of $75-100 \%$ for latent rheumatic heart disease and screening times of 2-4 minutes. ${ }^{26-28}$ However, few published studies have prospectively evaluated the real-world feasibility of single-view screening. One study from Sudan, showed that a single view protocol among 1400 children produced only four false positive screens and took an average of four minutes per exam. ${ }^{13}$ More recently, an augmented single-view screening protocol was prospectively evaluated in a cohort of school-children in Timor-Leste and demonstrated a sensitivity of $100 \%$ and a specificity of $95 \% .{ }^{29}$ However, rigorous studies have not evaluated the performance of simplified screening protocols on representative cohorts of at-risk children. Furthermore, how hand-held devices will perform for the detection of latent rheumatic heart disease has not been adequately explored. While there is increasing evidence that single view screening protocols can adequately detect latent rheumatic heart disease, there is no consensus on the criteria that define a positive scren. ${ }^{27,29,30}$ Due to inherent limitations in handheld ultrasound technology, criteria that perform well with one device cannot be assumed valid for another. To explore how a hand-held device compares to a fully equipped portable ultrasound device, we conducted a cross-sectional comparative study on a sample of schoolchildren to assess for differences between ultrasound devices when executing a single-view screening protocol. 


\section{Methods}

\section{Study Setting}

This cross-sectional study took place in November 2019 in Soddo, Ethiopia. Starting in April of 2019, Soddo Christian Hospital began operating a rheumatic heart disease screening program. The staff of Soddo Christian Hospital coordinate with local school leadership and perform school-based screenings 2-3 days weekly. The children are screened by grade level and gender. Any child found to have a positive screening ultrasound is referred to Soddo Christian Hospital for a confirmatory echocardiogram free of charge. If the confirmatory echocardiogram demonstrates definite or borderline rheumatic heart disease, the children and their guardian(s) are notified. A brief educational session is provided and the patient is provided ongoing monthly injections of penicillin. Six locally trained rheumatic heart disease screeners under the supervision of an onsite physician perform the screening ultrasounds. The screeners were recruited from a wide range of hospital staff including nurses, technicians, receptionists, and sanitation personnel. All screeners completed a mentored training program to become proficient in executing a single parasternal long-axis view of the heart with and without color-Doppler.

\section{Study Design}

Over a two-day period, our study team accompanied the screeners as they performed a school- based screening. On both study days, all children screened were between the ages of 15-18 and from the same school. For the screening, a portable Sonosite M-turbo ultrasound machine was used with a 5-1 Mhz phased array ultrasound probe. A Nyquist limit of $72 \mathrm{~cm} / \mathrm{s}$ was used for color Doppler images with a frame rate of $16.667 \mathrm{~Hz}$. All children determined to be positive by the screening team, as well as a random sample of children undergoing screening, were selected for our study. To minimize differences observed between devices, all children underwent repeat screening echocardiogram using the Sonosite Mturbo by a trained pediatric cardiac sonographer. This same echocardiographer then used a Phillips Lumify hand-held ultrasound device using a S4-1 Mhz phased array probe. For color Doppler imaging, the Lumify device has a fixed Nyquist limit of $60 \mathrm{~cm} / \mathrm{s}$ and an auto-adjusting frame rate. Two and three second video clips were stored on the Sonosite and Philips devices respectively. Images were then transferred onto encrypted flash drives and transferred onto secure hard drives for analysis. Clips contained a parasternal long-axis view of the heart with and without color Doppler. No associated demographic information was stored, and a random number was used to link the individual between devices. Where appropriate, separate images were saved for the aortic and mitral valves. Our study intervention did not alter the recommendations of the screening team and all children determined to be positive by the screening team were referred per protocol for confirmatory echocardiogram. This study was reviewed by the University of Minnesota Institutional Review Board and approved as a non-human research subject study.

\section{Study tool}


All identifying information was removed from the stored video clips. Due to obvious differences in image quality and clip duration, interpretation could not be blinded by device. All images were randomly arranged for analysis so that the interpreting study investigator was not able to compare images from one individual to another. All data entry occurred through REDCap. A 16-item interpretation survey was designed to capture the required elements for determining if the ultrasound was screen positive or screen negative. For the purposes of this study, a screening ultrasound was positive if the following criteria were met: 1. A pansystolic and multicolored regurgitation at the mitral valve by color Doppler estimated at a length of more than $1.5 \mathrm{~cm}$. If the regurgitation was eccentric, an estimated length of more than $1 \mathrm{~cm}$ was considered positive; 2 . Any regurgitation at the aortic valve; 3 . Any valvular abnormalities consistent with rheumatic heart disease. These criteria were derived from the findings of published studies and expert consensus. ${ }^{26,27,29}$ Our full survey tool can be reviewed in the supplemental materials. Two study investigators reviewed all ultrasounds, one an experienced cardiologist (R.J., expert) and the other an internal medicine and pediatric hospitalist (Z.K., non-expert) with experience in the use of point-of-care ultrasound. This design was used to capture differences in non-expert interpretation as might occur during routine school-based screenings.

\section{Statistical Analysis}

The primary objective was to determine the agreement between devices for each reader. For each reader, the agreement between devices was summarized and compared using McNemar's test for paired samples. Mitral valve (MV) and aortic valve (AV) abnormalities were summarized for positive agreement between devices, negative agreement between devices and disagreement between devices. To investigate the agreement in MV and $\mathrm{AV}$ abnormalities between devices for each of these three scenarios and for each reader, McNemar's test for paired samples was used. All reported p-values are two-sided and significance level of 0.05 was used. Statistical analyses were performed using R (version 3.6.1) and SAS (version 9.4, SAS Institute Inc., Cary, North Carolina).

\section{Results}

Nineteen children underwent ultrasounds with the M-turbo and Lumify devices. All ultrasounds contained adequate visualization of the mitral valve to assess for soft tissue and color Doppler abnormalities. All ultrasounds contained adequate imaging of the aortic valve. Four ultrasounds ( $3 \mathrm{M}$-turbo and 1 Lumify) contained inadequate color Doppler of the aortic valve by expert review. Expert interpretation demonstrated $84 \%$ agreement between the devices (Table 1). There was no association between screen status and the ultrasound device being used ( $p$-value 0.564). 
Table 1: Agreement between ultrasound devices for expert and non-expert review using a single-view parasternal long axis view of the heart with and without color Doppler

\section{Expert review.}

\section{Lumify screen positive Lumify screen negative $\quad P^{-v a l u e}{ }^{1}$}

$\begin{array}{llll}\text { M-Turbo screen positive } & 5(26.3 \%) & 1(5.3 \%) & 0.564 \\ \text { M-Turbo screen negative } & 2(10.5 \%) & 11(57.9 \%) & \end{array}$

${ }^{1} \mathrm{P}$-value is for McNemar's test for paired samples.

\section{Non-expert review.}

\section{Lumify screen positive Lumify screen negative $\quad P_{-v^{2}}{ }^{1}$}

$\begin{array}{llll}\text { M-Turbo screen positive } & 2(10.5 \%) & 3(15.8 \%) & 0.083\end{array}$

M-Turbo screen negative $0(0.0 \%) \quad 14(73.7 \%)$

${ }^{1} \mathrm{P}$-value is for McNemar's test for paired samples.

All disagreement was related to differences in the appearance of the mitral or aortic valves (Table 2). 
Table 2: Summary of disagreements between devices that determined screen positive versus screen negative status for expert and non-expert review.

\section{Expert Review*}

\begin{tabular}{|lll|}
\hline Variable & Lumify device $(\mathbf{N}=3)$ & M-Turbo device $(\mathbf{N}=3)$ \\
\hline MV chordal thickening, $n(\%)$ & $0(0.0 \%)$ & $1(33.3 \%)$ \\
\hline Yes & $3(100.0 \%)$ & $2(66.7 \%)$ \\
\hline No & & $1(33.3 \%)$ \\
\hline Any irregular or focal thickening of the AV, $n(\%)$ & $2(66.7 \%)$ & $2(66.7 \%)$ \\
\hline Yes & $1(33.3 \%)$ & \\
\hline
\end{tabular}

\section{Non-expert Review*}

\section{Variable}

Pan-systolic MV regurgitation, $\mathrm{n}(\%)$

Yes

No

Length of $\mathrm{MV}$ regurgitation jet, $\mathrm{n}(\%)$

$<1 \mathrm{~cm}$

$1-1.5 \mathrm{~cm}$

$1.5-2 \mathrm{~cm}$

$>2 \mathrm{~cm}$

Eccentric regurgitation jet, $\mathrm{n}(\%)$

Yes

No

Multi-colored mitral jet, $\mathrm{n}(\%)$

Yes

No

Any overlap/override of the anterior leaflet of the MV on the posterior leaflet during systole, $n(\%)$

Yes
Lumify device $(\mathrm{N}=3)$

$0(0.0 \%)$

$3(100.0 \%)$

$1(33.3 \%)$

$1(33.3 \%)$

1 (33.3\%)

$0(0.0 \%)$

$0(0.0 \%)$

3 (100.0\%)

3 (100.0\%)

$0(0.0 \%)$

1 (33.3\%)

3 (100.0\%)

$2(66.7 \%)$

$0(0.0 \%)$

M-Turbo device $(\mathrm{N}=3)$

3 (100.0\%)

$0(0.0 \%)$

$0(0.0 \%)$

$0(0.0 \%)$

2 (66.7\%)

1 (33.3\%) 
* Survey fields not shown did not have an effect on screen status. Please see supplemental tables for a review of all survey fields for disagreement by device.

Five children had screen positive ultrasounds with both devices. Four children screened positive with mitral regurgitation and one child had aortic regurgitation (Table 3). All jet lengths at the mitral valve for the Lumify were between $1-1.5 \mathrm{~cm}$. For the M-turbo, one jet length was $1.5-2 \mathrm{~cm}$ while the remaining were between 1-1.5 cm. Among the eleven children with screen negative ultrasounds on both devices, screennegative mitral regurgitation was significantly more common with the M-turbo (Supplemental tables). 
Table 3. Comparison of mitral valve and aortic valve abnormalities by ultrasound device for screen positive children by expert review.

\begin{tabular}{|c|c|c|c|}
\hline Variable & $\begin{array}{l}\text { Lumify } \\
\text { device } \\
(\mathrm{N}=5)\end{array}$ & $\begin{array}{l}\text { M-Turbo } \\
\text { device } \\
(\mathrm{N}=5)\end{array}$ & $\begin{array}{l}\mathrm{P}- \\
\text { value }\end{array}$ \\
\hline \multicolumn{3}{|l|}{ Adequately visualize the $\mathrm{MV}, \mathrm{n}(\%)$} & \multirow[t]{3}{*}{ N.E. } \\
\hline Yes & $\begin{array}{l}5 \\
(100.0 \%)\end{array}$ & $5(100.0 \%)$ & \\
\hline No & $0(0.0 \%)$ & $0(0.0 \%)$ & \\
\hline \multicolumn{3}{|l|}{ Any MV regurgitation, n (\%) } & \multirow[t]{3}{*}{ N.E. } \\
\hline Yes & $4(80.0 \%)$ & $4(80.0 \%)$ & \\
\hline No & $1(20.0 \%)$ & $1(20.0 \%)$ & \\
\hline \multicolumn{3}{|l|}{ Pan-systolic MV regurgitation, $\mathrm{n}(\%)$} & \multirow[t]{4}{*}{ N.E. } \\
\hline Number missing & 1 & 1 & \\
\hline Yes & $\begin{array}{l}4 \\
(100.0 \%)\end{array}$ & $4(100.0 \%)$ & \\
\hline No & $0(0.0 \%)$ & $0(0.0 \%)$ & \\
\hline \multicolumn{3}{|l|}{ Length of MV regurgitation jet, $\mathrm{n}(\%)$} & \multirow[t]{4}{*}{ N.E. } \\
\hline Number missing & 1 & 1 & \\
\hline $1-1.5 \mathrm{~cm}$ & $\begin{array}{l}4 \\
(100.0 \%)\end{array}$ & $3(75.0 \%)$ & \\
\hline $1.5-2 \mathrm{~cm}$ & $0(0.0 \%)$ & $1(25.0 \%)$ & \\
\hline \multicolumn{3}{|l|}{ Eccentric regurgitation jet, $\mathrm{n}(\%)$} & \multirow[t]{4}{*}{ N.E. } \\
\hline Number missing & 1 & 1 & \\
\hline Yes & $3(75.0 \%)$ & $4(100.0 \%)$ & \\
\hline No & $1(25.0 \%)$ & $0(0.0 \%)$ & \\
\hline \multicolumn{3}{|l|}{ Multi-colored mitral jet, n (\%) } & \multirow[t]{4}{*}{$>0.999$} \\
\hline Number missing & 1 & 1 & \\
\hline Yes & $3(75.0 \%)$ & $3(75.0 \%)$ & \\
\hline No & $1(25.0 \%)$ & $1(25.0 \%)$ & \\
\hline MV anterior leaflet thickening (>3mm), $\mathrm{n}(\%)$ & & & N.E. \\
\hline
\end{tabular}




\begin{tabular}{|c|c|c|c|}
\hline Yes & $2(40.0 \%)$ & $0(0.0 \%)$ & \\
\hline No & $3(60.0 \%)$ & $5(100.0 \%)$ & \\
\hline MV chordal thickening, $\mathrm{n}(\%)$ & & & N.E. \\
\hline Yes & $0(0.0 \%)$ & $1(20.0 \%)$ & \\
\hline No & $\begin{array}{l}5 \\
(100.0 \%)\end{array}$ & $4(80.0 \%)$ & \\
\hline Any restriction of the MV during diastole, $\mathrm{n}(\%)$ & & & N.E. \\
\hline Yes & $0(0.0 \%)$ & $0(0.0 \%)$ & \\
\hline No & $\begin{array}{l}5 \\
(100.0 \%)\end{array}$ & $5(100.0 \%)$ & \\
\hline $\begin{array}{l}\text { Any overlap/override of the anterior leaflet of the } \mathrm{MV} \text { on } \\
\text { the posterior leaflet during systole, } \mathrm{n}(\%)\end{array}$ & & & N.E. \\
\hline Yes & $0(0.0 \%)$ & $0(0.0 \%)$ & \\
\hline No & $\begin{array}{l}5 \\
(100.0 \%)\end{array}$ & $5(100.0 \%)$ & \\
\hline Adequate visualization of the $\mathrm{AV}, \mathrm{n}(\%)$ & & & N.E. \\
\hline Yes & $\begin{array}{l}5 \\
(100.0 \%)\end{array}$ & $5(100.0 \%)$ & \\
\hline No & $0(0.0 \%)$ & $0(0.0 \%)$ & \\
\hline Any $\mathrm{AV}$ regurgitation, $\mathrm{n}(\%)$ & & & N.E. \\
\hline Number missing & 1 & 1 & \\
\hline Yes & $1(25.0 \%)$ & $1(25.0 \%)$ & \\
\hline No & $3(75.0 \%)$ & $3(75.0 \%)$ & \\
\hline Any irregular or focal thickening of the $\mathrm{AV}, \mathrm{n}(\%)$ & & & 0.317 \\
\hline Yes & $2(50.0 \%)$ & $1(20.0 \%)$ & \\
\hline No & $2(50.0 \%)$ & $4(80.0 \%)$ & \\
\hline Any restriction in AV motion, $\mathrm{n}(\%)$ & & & N.E. \\
\hline Yes & $0(0.0 \%)$ & $0(0.0 \%)$ & \\
\hline No & $\begin{array}{l}5 \\
(100.0 \%)\end{array}$ & $5(100.0 \%)$ & \\
\hline Normal coaptation of the AV, $\mathrm{n}(\%)$ & & & N.E. \\
\hline Yes & $4(80.0 \%)$ & $5(100.0 \%)$ & \\
\hline
\end{tabular}




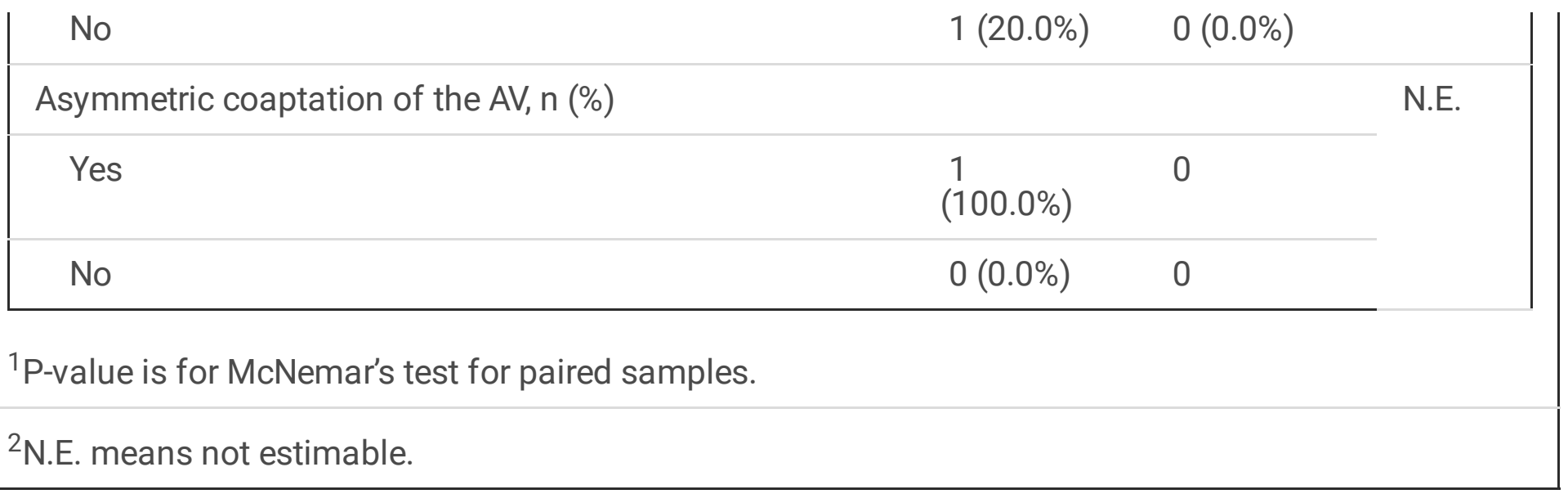

Non-expert interpretation was similar, showing $84 \%$ agreement between devices (Table 1). However, the M-turbo device was more likely to be associated with a positive screen and this trended towards statistical significance ( $p$-value 0.083). Disagreement was related to differences observed at the mitral valve (Table 2). Two children had screen positive ultrasounds by non-expert review on both devices. One child had pathological mitral regurgitation and one child had aortic regurgitation. Findings for these children were similar to expert review (supplemental tables). Among the 14 children with screen negative ultrasounds on both devices, screen-negative mitral regurgitation was more common with the M-turbo but did not reach statistical significance (Supplemental tables).

Among screen positive ultrasounds with abnormal mitral regurgitation, there was a trend in the M-turbo device towards longer estimated jet lengths by color Doppler when expert and non-expert interpretation was combined (Figure 1). When comparing non-expert to expert interpretation by device, the Lumify was more likely to be interpreted as screen negative ( $p$-value 0.025 , table 4 ). 
Table 4: Comparison of screen status by reviewer for each device.

\section{M-turbo}

\begin{tabular}{|llll|}
\hline & Non-expert review positive & Non-expert review negative & P-value $^{1}$ \\
Expert review positive & $5(26.3 \%)$ & $1(5.3 \%)$ & 0.317 \\
Expert review negative & $0(0.0 \%)$ & $13(68.4 \%)$ & \\
\cline { 1 - 3 }
\end{tabular}

${ }^{1} \mathrm{P}$-value is for McNemar's test for paired samples.

\section{Lumify}

\begin{tabular}{|llll|}
\hline & Non-expert review positive & Non-expert review negative & P-value $^{1}$ \\
Expert review positive & $2(10.5 \%)$ & $5(26.3 \%)$ & 0.025 \\
Expert review negative & $0(0.0 \%)$ & $12(63.2 \%)$ & \\
\hline
\end{tabular}

${ }^{1} \mathrm{P}$-value is for McNemar's test for paired samples.

We undertook a detailed evaluation of the three children in which there was discrepancy in screen status on the Lumify (Table 5). 
Table 5: Comparison of the mitral valve regurgitation in three children where there was disagreement in screen status by non-expert review.

\begin{tabular}{|lllll|}
\hline & Expert & \multicolumn{3}{l|}{ Non-expert } \\
Device & M-Turbo & Lumify ** & M-turbo & Lumify \\
Child 1 & & & & \\
Mitral jet length & $1-1.5 \mathrm{~cm}$ & $1-1.5 \mathrm{~cm}$ & $1.5-2 \mathrm{~cm}$ & $1.5-2 \mathrm{~cm}$ \\
Pansystolic & Yes & Yes & Yes & No \\
Eccentric & Yes & Yes & Yes & No \\
Multi-colored & No & Yes & Yes & Yes \\
Screen Positive & Yes & Yes & Yes & No \\
Child 2 & & & & \\
Mitral jet length & $1.5-2 \mathrm{~cm}$ & $1-1.5 \mathrm{~cm}$ & $>2 \mathrm{~cm}$ & $<1 \mathrm{~cm}$ \\
Pansystolic & Yes & Yes & Yes & No \\
Eccentric & Yes & Yes & Yes & No \\
Multicolored & Yes & Yes & Yes & No \\
Screen Positive & Yes & Yes & Yes & No \\
Child 3* & & & & Yes \\
Mitral jet length & $1-1.5 \mathrm{~cm}$ & $1-1.5 \mathrm{~cm}$ & $1.5-2 \mathrm{~cm}$ & $1-1.5 \mathrm{~cm}$ \\
Pansystolic & Yes & Yes & Yes & No \\
Eccentric & Yes & No & Yes & No \\
Mulicolored & Yes & No & Yes \\
Screen positive & Yes & Yes & Yes & No \\
\hline
\end{tabular}

* Child 3: On Lumify device, thickening was noted on the mitral valve but not seen on corresponding M-turbo image for expert review.

** Lumify: Color Doppler Frame rates varied per child: 1. 17 Hz., 2. 14 Hz., 3. $15 \mathrm{~Hz}$

There were important differences between expert and non-expert interpretation of the color Doppler images which impacted the screen status. Review of the observed mitral regurgitation in these children demonstrated more subtle color Doppler findings when viewed using the Lumify device (Figure 2).

\section{Discussion}


Among a small but representative sample of children at risk for rheumatic heart disease, our study demonstrates that there are important and measureable differences between two ultrasound devices, the handheld Philips Lumify and the Sonosite M-Turbo. When reviewed by an expert cardiologist, these differences were evenly split between devices and involved subtle differences in myocardial tissue imaging. When reviewed by a non-expert, the Lumify was more consistently associated with a screen negative interpretation. This difference trended towards statistical significance. When comparing screen status by device, there was a statistically significant difference between expert and non-expert interpretation using the Lumify. When images from the Lumify were reviewed by a non-expert, mitral jets were less likely to be categorized as pansystolic and estimated at a shorter length. Overall, mitral jets trended towards appearing shorter when viewed on the Lumify. Among screening ultrasounds that were categorized as negative, screen-negative mitral regurgitation was seen more commonly on the M-turbo.

Single-view screening protocols for the detection of latent rheumatic heart disease are increasingly demonstrating strong sensitivity and specificity. ${ }^{27,29}$ However, most studies to date have not evaluated handheld devices in a prospective manner. With the increasing availability and low cost of these devices, teams around the world have already started to employ modified versions of published single-view screening criteria. ${ }^{13}$ While these efforts are needed to help realize the potential of population based screening for rheumatic heart disease, our study demonstrates that there are important differences between ultrasound devices that may impact the sensitivity and specificity of single-view protocols especially when non-experts are interpreting the images. While expert review did not demonstrate perfect concordance between devices, the disagreement was isolated to myocardial tissue imaging abnormalities. These discordant findings highlight the importance of myocardial imaging optimization during screening protocols. For example, one discrepancy between the devices occurred because the midfield gain was too low on the M-turbo. This led to an inability to detect the valvular abnormality, even on expert review. However, prior research demonstrates that follow-up of screen positive ultrasounds for isolated valvular abnormalities are most likely borderline by World Heart Federation criteria and much less likely to progress on follow-up echocardiograms. ${ }^{30-34}$

Our study has many important limitations. While all images were captured by the same experienced pediatric cardiac sonographer, differences in image acquisition may explain some of the differences observed. Some differences are expected between expert and non-expert interpretation of echocardiograms for rheumatic heart disease screening. ${ }^{22,24,35,36}$ However, it is notable that agreement regarding screen status on the M-turbo device was extremely high between reviewers with no case of screen-positive valvular regurgitation being missed by non-expert review suggesting that differences in appearance between the ultrasound devices explains the discordance in screen status. Given the importance of mitral regurgitation in the early detection of rheumatic heart disease, this trend when evaluated over a larger sample of children, could have significant impacts.

There are also inherent differences between the devices. The Phillips Lumify has a fixed Nyquist limit of $60 \mathrm{~cm} / \mathrm{s}$ whereas the Sonosite M-turbo can be adjusted. For the purposes of this study, the Nyquist limit on the M-turbo was set a $72 \mathrm{~cm} / \mathrm{s}$. The Nyquist limit on the M-turbo was not adjusted to minimize 
interruptions to the standard imaging settings already employed by the screening team. Interestingly, our observed trend towards lower jet lengths in the Lumify are not readily explained by the lower Nyquist limit as one would expect increased aliasing and artifact given the high velocities being observed. ${ }^{37}$ Reducing the Nyquist limit in the M-turbo would have exacerbated the observed discrepancies in jet length which suggest the jet length discrepancy is related to other factors. There are also differences in the frame rate between devices. Review of the color Doppler and myocardial images from the M-turbo show a fixed frame rate of $16.667 \mathrm{hz}$. In contrast, the Lumify device automatically adjusts the frame rate depending on several factors. In our study, the frame rate ranged from $14-17 \mathrm{~Hz}$ during color Doppler acquisition but was much higher, $29-32 \mathrm{~Hz}$, during myocardial imaging. It is possible that a lower frame rate could result in a shorter jet length by color Doppler. On a per second basis, there was a maximum of approximately two fewer frames in the Lumify device. These frame rates are very comparable and unlikely to explain more than trivial differences in jet length or duration. While the frame rates during myocardial imaging are much more substantial, the impacts of this were not apparent on our analysis but may have been limited by the small sample size. Our study sample was too small to evaluate for the impact of the higher frame rates on the Lumify during myocardial imaging.

Single-view screening protocols need to weigh the balance between sensitivity and specificity. The differences observed in our study suggest that the thresholds used to determine the ideal positive screen may be influenced by unique and unpredictable performance characteristics of the ultrasound device being utilized. Few other studies have directly compared the imaging characteristics of handheld ultrasound devices to a fully equipped device in a real world-screening environment. Our study highlights that the ideal criteria for determining a screen positive ultrasound may vary by ultrasound device. This may be more important among screening programs that utilize non-expert ultrasonographers to carry out school-based screenings. Criteria that move away from length based estimations of color Doppler jets and focus on other characteristics of pathological regurgitation seen in latent rheumatic heart disease may be preferable. ${ }^{29}$ Ongoing research efforts examining the Philips Lumify device are underway and may help shed light on the appropriate criteria for single-view screening protocols and handheld ultrasound devices. ${ }^{38}$ Until further evidence is available, the use of handheld devices for rheumatic heart disease screening should be approached cautiously to avoid missing cases that would otherwise be referred for confirmatory echocardiograms.

\section{Declarations}

\section{Acknowledgments:}

We acknowledge the hard work of the rheumatic heart disease screening team and support staff of Soddo Christian Hospital.

\section{Funding:}


Zachary P. Kaltenborn received Funding for this project from the University of Minnesota Center for Global Health and Social Responsibility Faculty Travel Awards Program

Jonathan D Kirsch obtained the Philips Lumify used in this study through the University of Minnesota Foundation, Faculty Research and Equipment Grant, 2018

Research reported in this publication was supported by NIH grant P30 CA77598 utilizing the Biostatistics and Bioinformatics Core shared resource of the Masonic Cancer Center, University of Minnesota and by the National Center for Advancing Translational Sciences of the National Institutes of Health Award Number UL1TR002494. The content is solely the responsibility of the authors and does not necessarily represent the official views of the National Institutes of Health.

\section{Conflicts of Interest:}

All authors have reviewed the above manuscript and attest that they have no financial or non-financial conflicts of interest.

\section{Ethical Approval and Consent to Participate:}

This study was reviewed and approved by the Institutional Review Board at the University of Minnesota and determined to be a non-human research subjects study (STUDY00008308). The IRB determined that the proposed activity is not research involving human subjects as defined by DHHS and FDA regulations. The rheumatic heart disease screening program is done in cooperation with local educational and health ministries. The consent process for participation in the screening program is described above and was not altered by this study.

\section{Availability of data and materials:}

All data generated or analysed during this study are included in this published article and its supplementary information files

\section{Consent for publication}

Not applicable. Published image is fully de-identified.

\section{Authors' contributions}

ZPK: Contributed to this study through completion of IRB, obtaining study images, non-expert review of images, data analysis, and manuscript preparation.

AZ: Contributed to this study through study design and manuscript review

JDK: Contributed to this study through study design, providing the Philips Lumify device, and manuscript review 
MY: Contributed to this study through onsite support for screening staff, study design, image acquisition, and manuscript review.

KTM: Contributed to this study through statistical analysis and manuscript review

EN: Contributed to this study through study design, ultrasound image acquisition, image validation, sonographic expertise, and manuscript review

RAJ: Contributed to this study through screening team training, study design, image acquisition, image analysis (expert), data analysis, and manuscript review.

\section{References}

1. Watkins DA, Johnson CO, Colquhoun SM, et al. Global, Regional, and National Burden of Rheumatic Heart Disease, 1990-2015. N Engl J Med. 08 2017;377(8):713-722. doi:10.1056/NEJMoa1603693

2. Watkins $D$, Daskalakis A. The economic impact of rheumatic heart disease in developing countries. Consortium of Universities for Global Health, 6th Annual Conference: Lancet Global Health; 2015. p. S37.

3. Ordunez P, Martinez R, Soliz P, Giraldo G, Mujica OJ, Nordet P. Rheumatic heart disease burden, trends, and inequalities in the Americas, 1990-2017: a population-based study. Lancet Glob Health. 10 2019;7(10):e1388-e1397. doi:10.1016/S2214-109X(19)30360-2

4. Carapetis JR, Steer AC, Mulholland EK, Weber M. The global burden of group A streptococcal diseases. Lancet Infect Dis. Nov 2005;5(11):685-94. doi:10.1016/S1473-3099(05)70267-X

5. FEINSTEIN AR, SPAGNUOLO M, WOOD HF, TARANTA A, TURSKY E, KLEINBERG E. RHEUMATIC FEVER IN CHILDREN AND ADOLESCENTS. A LONG-TERM EPIDEMIOLOGIC STUDY OF SUBSEQUENT PROPHYLAXIS, STREPTOCOCCAL INFECTIONS, AND CLINICAL SEQUELAE. VI. CLINICAL FEATURES OF STREPTOCOCCAL INFECTIONS AND RHEUMATIC RECURRENCES. Ann Intern Med. Feb 1964;60:SUPPL 5:68-86.

6. Tompkins DG, Boxerbaum B, Liebman J. Long-term prognosis of rheumatic fever patients receiving regular intramuscular benzathine penicillin. Circulation. Mar 1972;45(3):543-51.

7. Grover A, Dhawan A, lyengar SD, Anand IS, Wahi PL, Ganguly NK. Epidemiology of rheumatic fever and rheumatic heart disease in a rural community in northern India. Bull World Health Organ. 1993;71(1):59-66.

8. Kassem AS, el-Walili TM, Zaher SR, Ayman M. Reversibility of mitral regurgitation following rheumatic fever: clinical profile and echocardiographic evaluation. Indian J Pediatr. 1995 Nov-Dec 1995;62(6):717-23.

9. Marijon E, Ou P, Celermajer DS, et al. Prevalence of rheumatic heart disease detected by echocardiographic screening. N Engl J Med. Aug 2007;357(5):470-6. doi:10.1056/NEJMoa065085

10. Rothenbühler M, O'Sullivan CJ, Stortecky S, et al. Active surveillance for rheumatic heart disease in endemic regions: a systematic review and meta-analysis of prevalence among children and 
adolescents. Lancet Glob Health. Dec 2014;2(12):e717-26. doi:10.1016/S2214-109X(14)70310-9

11. Reményi B, Wilson N, Steer A, et al. World Heart Federation criteria for echocardiographic diagnosis of rheumatic heart disease-an evidence-based guideline. Nat Rev Cardiol. Feb 2012;9(5):297-309. doi:10.1038/nrcardio.2012.7

12. Yadeta D, Hailu A, Haileamlak A, et al. Prevalence of rheumatic heart disease among school children in Ethiopia: A multisite echocardiography-based screening. Int J Cardiol. Oct 2016;221:260-3. doi:10.1016/j.ijcard.2016.06.232

13. Ali S, Domi S, Abbo B, et al. Echocardiographic screening for rheumatic heart disease in 4515 Sudanese school children: marked disparity between two communities. Cardiovasc J Afr. 2018 Sep/Oct 23 2018;29(5):273-277. doi:10.5830/CVJA-2018-022

14. Beaton A, Okello E, Lwabi P, Mondo C, McCarter R, Sable C. Echocardiography screening for rheumatic heart disease in Ugandan schoolchildren. Circulation. Jun 2012;125(25):3127-32. doi:10.1161/CIRCULATIONAHA.112.092312

15. Kane A, Mirabel M, Touré K, et al. Echocardiographic screening for rheumatic heart disease: age matters. Int J Cardiol. Sep 2013;168(2):888-91. doi:10.1016/j.ijcard.2012.10.090

16. Sims Sanyahumbi A, Sable CA, Beaton A, et al. School and Community Screening Shows Malawi, Africa, to Have a High Prevalence of Latent Rheumatic Heart Disease. Congenit Heart Dis. Dec 2016;11(6):615-621. doi:10.1111/chd.12353

17. Hunter LD, Monaghan M, Lloyd G, Pecoraro AJK, Doubell AF, Herbst PG. Screening for rheumatic heart disease: is a paradigm shift required? Echo Res Pract. Dec 2017;4(4):R43-R52. doi:10.1530/ERP-17-0037

18. Dougherty S, Khorsandi M, Herbst P. Rheumatic heart disease screening: Current concepts and challenges. Ann Pediatr Cardiol. 2017 Jan-Apr 2017;10(1):39-49. doi:10.4103/0974-2069.197051

19. Beaton A, Lu JC, Aliku T, et al. The utility of handheld echocardiography for early rheumatic heart disease diagnosis: a field study. Eur Heart J Cardiovasc Imaging. May 2015;16(5):475-82. doi:10.1093/ehjci/jeu296

20. Carapetis JR, Zühlke LJ. Global research priorities in rheumatic fever and rheumatic heart disease. Ann Pediatr Cardiol. Jan 2011;4(1):4-12. doi:10.4103/0974-2069.79616

21. Campbell J DG, Buchan J, Pozo-Martin F, Guerra Arias M, Leone C, Siyam A, Cometto G. A universal truth: no health without a workforce. 2013.

22. Ploutz M, Lu JC, Scheel J, et al. Handheld echocardiographic screening for rheumatic heart disease by non-experts. Heart. Jan 2016;102(1):35-9. doi:10.1136/heartjnl-2015-308236

23. Mirabel M, Bacquelin R, Tafflet M, et al. Screening for rheumatic heart disease: evaluation of a focused cardiac ultrasound approach. Circ Cardiovasc Imaging. Jan 2015;8(1)doi:10.1161/CIRCIMAGING.114.002324

24. Engelman D, Kado JH, Reményi B, et al. Focused cardiac ultrasound screening for rheumatic heart disease by briefly trained health workers: a study of diagnostic accuracy. Lancet Glob Health. 06 2016;4(6):e386-94. doi:10.1016/S2214-109X(16)30065-1 
25. Beaton A, Nascimento BR, Diamantino AC, et al. Efficacy of a Standardized Computer-Based Training Curriculum to Teach Echocardiographic Identification of Rheumatic Heart Disease to Nonexpert Users. Am J Cardiol. 06 2016;117(11):1783-9. doi:10.1016/j.amjcard.2016.03.006

26. Zühlke LJ, Engel ME, Nkepu S, Mayosi BM. Evaluation of a focussed protocol for hand-held echocardiography and computer-assisted auscultation in detecting latent rheumatic heart disease in scholars. Cardiol Young. Aug 2016;26(6):1097-106. doi:10.1017/S1047951115001857

27. Diamantino A, Beaton A, Aliku T, et al. A focussed single-view hand-held echocardiography protocol for the detection of rheumatic heart disease. Cardiol Young. 01 2018;28(1):108-117. doi:10.1017/S1047951117001676

28. Lu JC, Sable C, Ensing GJ, et al. Simplified rheumatic heart disease screening criteria for handheld echocardiography. J Am Soc Echocardiogr. Apr 2015;28(4):463-9. doi:10.1016/j.echo.2015.01.001

29. Remenyi B, Davis K, Draper A, et al. Single Parasternal-Long-Axis-View-Sweep Screening Echocardiographic Protocol to Detect Rheumatic Heart Disease: A Prospective Study of Diagnostic Accuracy. Heart Lung Circ. Jun 2019;doi:10.1016/j.hlc.2019.02.196

30. Nunes MCP, Sable C, Nascimento BR, et al. Simplified Echocardiography Screening Criteria for Diagnosing and Predicting Progression of Latent Rheumatic Heart Disease. Circ Cardiovasc Imaging. Feb 2019;12(2):e007928. doi:10.1161/CIRCIMAGING.118.007928

31. Beaton A, Aliku T, Dewyer A, et al. Latent Rheumatic Heart Disease: Identifying the Children at Highest Risk of Unfavorable Outcome. Circulation. Dec 2017;136(23):2233-2244. doi:10.1161/CIRCULATIONAHA.117.029936

32. Beaton A, Okello E, Aliku T, et al. Latent rheumatic heart disease: outcomes 2 years after echocardiographic detection. Pediatr Cardiol. Oct 2014;35(7):1259-67. doi:10.1007/s00246-0140925-3

33. Zühlke L, Engel ME, Lemmer CE, et al. The natural history of latent rheumatic heart disease in a 5 year follow-up study: a prospective observational study. BMC Cardiovasc Disord. Feb 2016;16:46. doi:10.1186/s12872-016-0225-3

34. Engelman D, Wheaton GR, Mataika RL, et al. Screening-detected rheumatic heart disease can progress to severe disease. Heart Asia. 2016;8(2):67-73. doi:10.1136/heartasia-2016-010847

35. Nascimento BR, Beaton AZ, Nunes MC, et al. Echocardiographic prevalence of rheumatic heart disease in Brazilian schoolchildren: Data from the PROVAR study. Int J Cardiol. Sep 2016;219:43945. doi:10.1016/j.ijcard.2016.06.088

36. Engelman D, Kado JH, Reményi B, et al. Screening for rheumatic heart disease: quality and agreement of focused cardiac ultrasound by briefly trained health workers. BMC Cardiovasc Disord. Feb 2016;16:30. doi:10.1186/s12872-016-0205-7

37. McCulloch M, Cleve J. Echocardiography. Chapter 3: Machine Controls and Optimizing images. Second ed. Springer, Cham; 2018.

38. Francis JR, Fairhurst $H$, Whalley $G$, et al. The RECARDINA Study protocol: diagnostic utility of ultraabbreviated echocardiographic protocol for handheld machines used by non-experts to detect 
rheumatic heart disease. BMJ Open. 05 2020;10(5):e037609. doi:10.1136/bmjopen-2020-037609

\section{Figures}

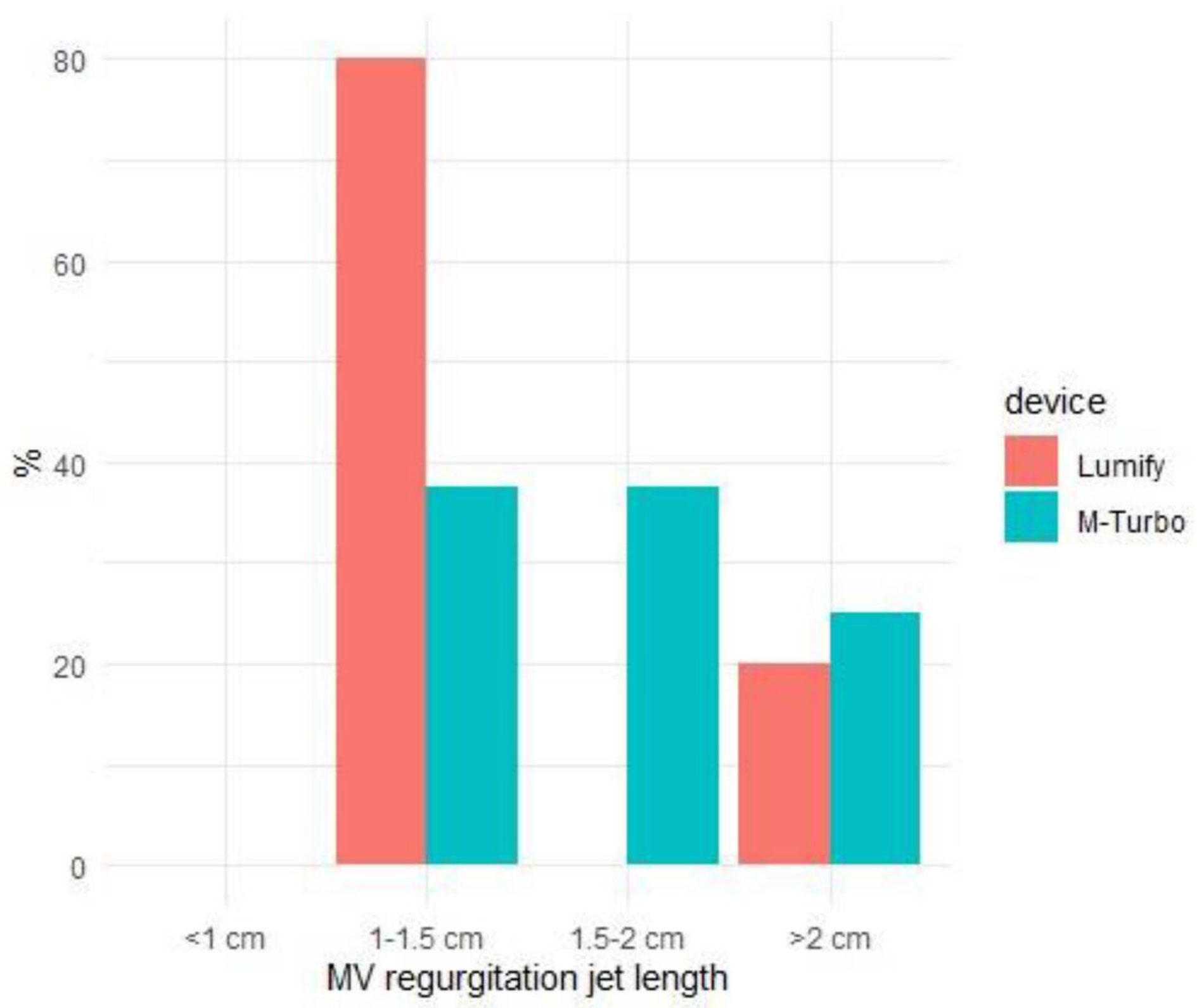

\section{Figure 1}

Title: Estimated length of mitral regurgitation for both both reviewers by device Legend: Aggregate estimated length of mitral regurgitation jets by color doppler for all children for both reviewers that had pansystolic mitral regurgitation and had a positive screen 


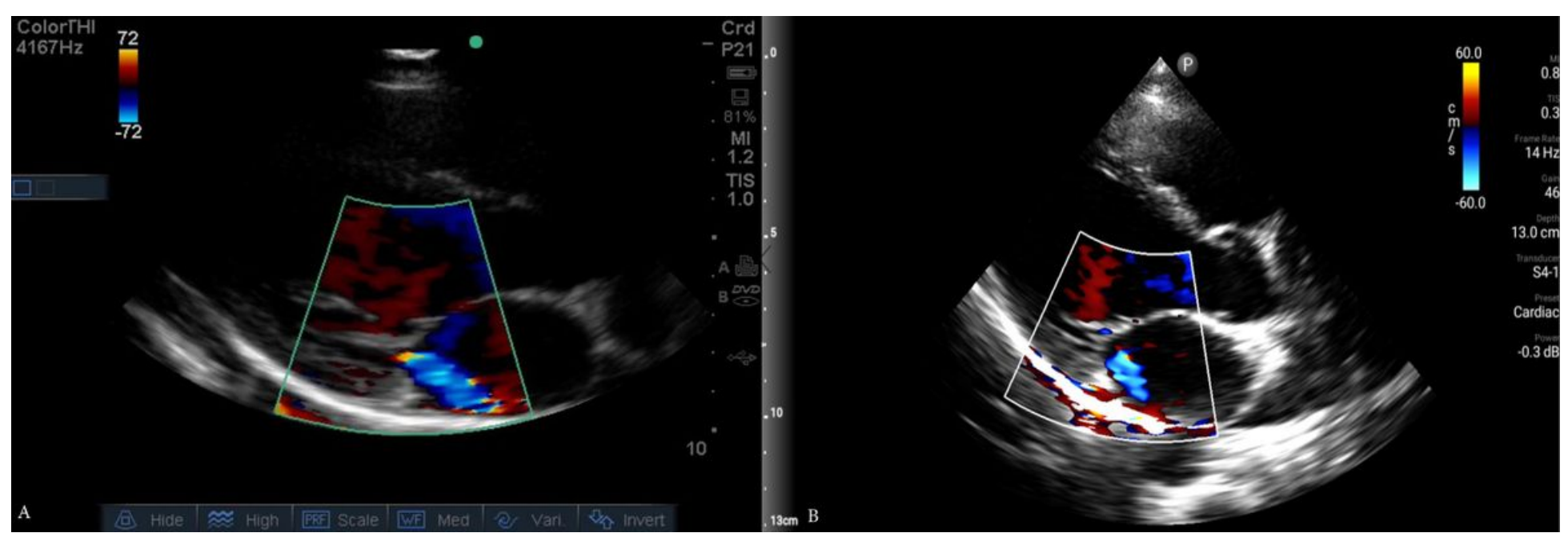

\section{Figure 2}

Title: Comparison of color Doppler images between the M-turbo and Lumify Legend: Side by Sde images from the same child illustrating the differential appearance of mitral regurgitation by color Doppler between the M-turbo (A) and the Lumify (B). These images represent the most abnormal jet from the respective video clips as determined by expert review.

\section{Supplementary Files}

This is a list of supplementary files associated with this preprint. Click to download.

- SupplementalTables.docx 